\title{
Two-dimensional layered architecture constructing energy and phonon blocks for enhancing thermoelectric performance of InSb
}

\author{
Jiwu Xin ${ }^{1}$, Wang $\mathrm{Li}^{1}$, Sihui $\mathrm{Li}^{1}$, Yang $\mathrm{Tao}^{1}$, Tian $\mathrm{Xu}^{1}$, Yubo Luo ${ }^{1 *}$, Qinghui Jiang ${ }^{1}$, Lei $\mathrm{Wei}^{2^{*}}$ and \\ Junyou Yang ${ }^{1 *}$
}

\begin{abstract}
InSb is a narrow-bandgap semiconductor with a zinc blende structure and has been wildly applied in photodetectors, infrared thermal imaging, and Hall devices. The facts of decent band structure, ultrahigh electron mobility, and nontoxic nature indicate that InSb may be a potential mid-temperature thermoelectric material. The critical challenges of InSb, such as high thermal conductivity and small Seebeck coefficient, have induced its ultrahigh lattice thermal conductivity, and thus low $Z T$ values. In view of this, we have developed a competitive strategy typified by the cost-efficient nanocompositing of $z \mathrm{wt} \% \mathrm{QSe}_{2}(\mathrm{Q}=\mathrm{Sn}, \mathrm{W})$. Specifically, the $\mathrm{Q}_{\mathrm{In}}{ }^{+}$and $\mathrm{Se}_{\mathrm{Sb}}{ }^{+}$point defects were introduced in the InSb system by nanocompositing the vested two-dimensional layered $\mathrm{QSe}_{2}$. In addition, the enlarged valence band maximum of intrinsic $\mathrm{WSe}_{2}$ acted as ladders can scatter a fair number of hole carriers, resulting in the relatively enhanced Seebeck coefficient of high temperature. Moreover, the disorderly distributed nanosheets/particles, and dislocations acting as obstacles can effectively delay the heat flow diffusion, inducing the strong scattering of thermal phonons. Consequently, an enhanced power factor of $\sim 33.3 \mu \mathrm{W} \mathrm{cm} \mathrm{cm}^{-1} \mathrm{~K}^{-2}$ and $Z T$ value of $\sim 0.82$ at $733 \mathrm{~K}$ have been achieved in the $3 \% \mathrm{WSe}_{2}$ sample, companied with the engineering output power density $\omega_{\max }$ $\sim 233 \mu \mathrm{W} \mathrm{cm}$ and thermoelectric conversion efficiency $\eta$ $\sim 5.2 \%$. This artificially designed approach indicated by suited nanocompositing can integrate several engineering strategies such as point defects, nanoengineering, and energy filtering into one, providing a reference to optimize the thermoelectric performance of other thermoelectric systems.
\end{abstract}

Keywords: thermoelectric, InSb, energy barrier, $\mathrm{WSe}_{2}$ nanosheets, output power density

\section{INTRODUCTION}

Thermoelectric generators (TEGs) can convert the industrial waste heat into electricity, which is of great significance to alleviate the energy crisis and the greenhouse effect [1-4]. For generating electricity through the thermoelectric effect, a thermoelectric module and a temperature difference between its hot and cold side are necessary. The most commonly used commercial thermoelectric materials are $\mathrm{SiGe}[5], \mathrm{Bi}_{2} \mathrm{Te}_{3}$ [6] and $\mathrm{PbTe}$ [7], and recently, some new materials such as half-Heusler
[8] and SnSe [9] have been demonstrated to have a better efficiency but require higher temperature difference. The conversion efficiency of thermoelectric devices is closely related with the $Z T=S^{2} s T / k$ of TE materials, where $S, s, k, T$ are the Seebeck coefficient, electrical conductivity, thermal conductivity, and absolute temperature, respectively. Among them, $k=k_{\mathrm{e}}+k_{\mathrm{L}}$ is derived from the carrier thermal conductivity $\left(k_{\mathrm{e}}\right)$ and lattice thermal conductivity $\left(k_{\mathrm{L}}\right)$ [10-12]. The classic theory of phonon glass electron crystal (PGEC) provides a route to design a high$Z T$ thermoelectric material with higher electrical performance and low thermal conductivity. Therefore, numerous separate approaches have been devoted to optimizing the electrical and thermal properties, thus increasing the $Z T$ value, and the electrical performance can be optimized via point defects (PDs) $[13,14]$, band engineering $[15,16]$, and high-entropy engineering $[17,18]$. In contrast, the microstructure engineering induced by nanocompositing $[19,20]$, dislocations [21,22], and hierarchical architecture [23,24], has been employed and proven to be effective in reducing the thermal conductivity.

InSb is a narrow-bandgap $(\sim 0.18 \mathrm{eV})$ semiconductor with a zinc blende structure, and its characteristics of high electron mobility $\left(\sim 78,000 \mathrm{~cm}^{2} \mathrm{~V}^{-1} \mathrm{~s}^{-1}\right)[25,26]$ and $\mathrm{Pb}$-free composition indicate that it may be a promising mid-temperature thermoelectric material. Numerous effects have been employed to improve the thermoelectric properties of InSb in recent years. For instance, Su et al. [27] obtained a ZT value of $\sim 0.49$ at $700 \mathrm{~K}$ for polycrystalline InSb by a Melt-MS-SPS (MS: melt spinning; SPS: spark plasma sintering) process. Zhang et al. [28] reported an improved $Z T$ value of $\sim 0.73$ at $650 \mathrm{~K}$ in a Ga-doped $\mathrm{InSb}$ compound. Jiang et al. [29] got a decreased lattice thermal conductivity of $\sim 10 \mathrm{Wm}^{-1} \mathrm{~K}^{-1}$ in InSb via in situ introducing $\mathrm{NiSb}$ precipitates and more recently, Yang and co-workers $[30,31]$ achieved a large reduction of lattice thermal conductivity in a narrow temperature range with the help of InSb-Sb eutectic structure. However, the thermoelectric performance of InSb is far inferior to the currently mid-temperature thermoelectric materials such as $\mathrm{PbTe}$ [32] and $\mathrm{CoSb}_{3}$ [33], which is mainly due to its high thermal conductivity and small Seebeck coefficient. As is known, the thermal conductivity of TE materials can be largely reduced through introducing multi-scale second phases, and the electrical transport properties such as carrier mobility may also get deteriorated. Meanwhile, inducing PDs via singleor co-doping is an effective approach to increase the carrier

\footnotetext{
${ }^{1}$ State Key Laboratory of Materials Processing and Die and Mould Technology, Huazhong University of Science and Technology, Wuhan 430074, China

${ }^{2}$ School of Electrical and Electronic Engineering, Nanyang Technological University, 50 Nanyang Avenue, Singapore 639798, Singapore

* Corresponding authors (emails: jyyang@mail.hust.edu.cn (Yang J); wei.lei@ntu.edu.sg (Wei L); luoyubo@hust.edu.cn (Luo Y))
} 
concentration, except for the decreased Seebeck coefficient due to the Mott relationship [34].

As is known, $\mathrm{WSe}_{2}$ and $\mathrm{SnSe}_{2}$ are emerging two-dimensional layered compounds with ultra-low thermal conductivities, and their partial elements of anion and cation sites may be dissolved into the InSb crystal lattice during hot pressing. In this scenario, we develop a merged strategy for regulating the electrical and thermal properties via subtly building thermal and energy barriers, which can be detailed by the nanocompositing of $z$ wt\% $\mathrm{QSe}_{2}(\mathrm{Q}=\mathrm{Sn}, \mathrm{W})$ with the InSb system. Benefiting from it, the enhanced electrical conductivity originated from the generated $\mathrm{Q}_{\mathrm{In}}{ }^{+}$and $\mathrm{Se}_{\mathrm{Sb}}{ }^{+}$PDs. Meanwhile, the suitable energy ladder constructed by the valence band maximum between the InSb and $\mathrm{WSe}_{2}$ can effectively scatter low energy carriers, thus leading to an enhanced Seebeck coefficient compared with $\mathrm{SnSe}_{2}$-composited sample. As a result, a $43.2 \%$ increased power factor of $33.3 \mu \mathrm{W} \mathrm{cm} \mathrm{cm}^{-1} \mathrm{~K}^{-2}$ has been achieved in the $3 \% \mathrm{WSe}_{2}$ sample. Moreover, the hierarchical microstructure such as nanosheets/ particles, abundant dislocations, and strain fields can effectively scatter multi-scale phonons, leading to a decreased lattice thermal conductivity of 1.1 and $1.02 \mathrm{~W} \mathrm{~m}^{-1} \mathrm{~K}^{-2}$ with the additives of 9\% $\mathrm{SnSe}_{2}$ and $3 \% \mathrm{WSe}_{2}$, respectively. Ultimately, an enlarged thermoelectric performance of $Z T$ value $\sim 0.82$ at $733 \mathrm{~K}$ has been achieved in the $3 \% \mathrm{WSe}_{2}$-composited sample, which results in $30 \%$ and $134 \%$ improvement compared with the $\mathrm{SnSe}_{2}$ composites $(Z T \sim 0.63)$ and pristine InSb $(Z T \sim 0.35)$. This work realizes multiple functions of PDs, energy filtering, and microstructure engineering via cost-efficient $\mathrm{QSe}_{2}$ nanocompositing, synchronously optimizing the electrical and thermal properties of InSb, which could be taken as an example for other high thermal conductivity thermoelectric systems.

\section{EXPERIMENTAL SECTION}

\section{Fabrication of thermoelectric materials}

Indium powder (99.99\%, Changsha Asian Light Co., Ltd.) and antimony powder (99.99\%, Changsha Asian Light Co., Ltd.) were mixed in an exact metage according to nominal composition $\mathrm{In}_{1.01} \mathrm{Sb}$, slowly heated to $973 \mathrm{~K}$ at a rate of $117 \mathrm{~K} \mathrm{~h}^{-1}$ and held at this temperature for $6 \mathrm{~h}$. Then the InSb ingots were ground into fine powders in an agate mortar. As for the preparation of $\mathrm{QSe}_{2}(\mathrm{Q}=\mathrm{Sn}, \mathrm{W})$, the acquisition of $\mathrm{SnSe}_{2}$ was referred to the previous work [35], and the $\mathrm{WSe}_{2}$ was obtained through the melting-quenching method [36]. The $\mathrm{QSe}_{2}$ ingot was ground into uniform powder and performed in an ultrasonic vibrator for $6 \mathrm{~h}$ to obtain $\mathrm{QSe}_{2}$ nanopowders. Subsequently, the prepared $\mathrm{QSe}_{2}$ was mixed with the sieved pristine InSb well and then moved to a graphite mold for SPS sintering, consolidated at $733 \mathrm{~K}$ for $10 \mathrm{~min}$ in a graphite mold $(20 \mathrm{~mm}$ in diameter) with a compressive stress of $60 \mathrm{MPa}$. All the cylinders were obtained and characterized with relative densities at least 95\%.

\section{DFT calculated details}

The density functional theory (DFT) was implemented in the Vienna $a b$ initio simulation package (VASP) [37]. The geometry of system is fully relaxed until the magnitude of the force acting on all atoms is less than $10^{-5} \mathrm{eV} \AA^{-1}$ with a kinetic energy cutoff of $520 \mathrm{eV}$. The $2 \times 2 \times 2$ supercells with the Gamma centered kpoint grid of $6 \times 6 \times 6(\mathrm{InSb})$ and $9 \times 9 \times 2\left(\mathrm{WSe}_{2}\right)$ were adopted for the Brillouin zone to calculate the total energy and density of states (DOS), respectively.

\section{Characterizations of phases and microstructures}

The powder X-ray diffraction (PXRD) was characterized on a PANalytical Empyrean diffractometer. The in situ PXRD was performed on Rigaku Smartlab equipped with $\mathrm{Cu} \mathrm{Ka}(\lambda=$ $1.5418 \AA$ ). The electron probe micro-analysis (EPMA) measurements were carried out on the EPMA-8050G instruments, in which the analysis accuracy of the element content is less than $5 \%$. The high-resolution transmission electron microscopy (HRTEM), energy-dispersive X-ray spectroscopy (EDS), and high-angle annular dark-field (HAADF) analysis of sheared InSb specimen were completed by the TEM observation performed on a Talos F200X microscope equipped with corresponding advanced accessories.

\section{Measurements of thermoelectric properties}

The temperature-dependent Hall coefficient $\left(R_{\mathrm{H}}\right)$ was obtained by using the van der Pauw technique under a reversible magnetic field $(\sim 1.5 \mathrm{~T})$ [38]; the Hall carrier concentration $\left(p_{\mathrm{H}}\right)$ and Hall mobility $\left(\mu_{\mathrm{H}}\right)$ were then calculated by the equation of $n_{\mathrm{H}}=$ $1 /\left(e R_{\mathrm{H}}\right)$ and $\mu_{\mathrm{H}}=R_{\mathrm{H}} / \rho$, respectively. The Seebeck coefficient and electrical resistivity were measured by a commercial thermoelectric measurement system (ZEM-3, ULVAC). The longitudinal $\left(v_{\mathrm{L}}\right)$ and transverse $\left(v_{\mathrm{T}}\right)$ velocities were obtained from the measurement based on the OLYMPUS model 5072PR. The relative densities ( $d$, refer to Table $S 1)$ and thermal diffusivity $(D)$ of bulk samples were measured by the Archimedes method and laser flash method (LFA 427, NETZSCH); heat capacity $\left(C_{\mathrm{p}}\right)$ was derived from the Debye model (Supplementary information Note), and thus the thermal conductivity $(\kappa)$ was derived from $\kappa$ $=d D C_{\mathrm{p}}$. The deviation of $Z T$ values was considered as $20 \%$ [39], which can be detailed explicitly by the uncertainty of $10 \%, 5 \%$, and $3 \%$ of thermal conductivity, Seebeck coefficient, and electrical resistivity, respectively.

\section{RESULTS AND DISCUSSION}

\section{Phase characterizations and microstructure observations}

Fig. 1 depicts the XRD patterns of $\mathrm{InSb}+z \mathrm{wt} \% \mathrm{QSe}_{2}$-composited samples detailed by the $z$ wt $\% \operatorname{SnSe}_{2}(z=0,3,6,9)$ and $z$ wt $\% \mathrm{WSe}_{2}(z=0,3,6,9)$. Except for the well-matched main diffraction peaks of zinc-blende InSb, traces of second phases such as Se (PDF\#54-0500), and SnSb (PDF\#33-0118) were observed in the $\mathrm{SnSe}_{2}$ composites as shown in Fig. 1a. The intensity of $\mathrm{WSe}_{2}$ phase (PDF\#71-0600) was increased with increasing concentration of $z \mathrm{wt} \%$ (Fig. 1b). Especially, the PXRD analysis displayed in Fig. S1 confirms that the pure $\mathrm{SnSe}_{2}$ and $\mathrm{WSe}_{2}$ powders had been obtained, which clearly reveals that the $\mathrm{WSe}_{2}$ is compatible with the InSb matrix while the $\mathrm{SnSe}_{2}$ is not. According to the principle of lowest energy, the formation energy/atom of $\mathrm{SnSe}_{2}$ and $\mathrm{WSe}_{2}$ is -0.345 and $-0.558 \mathrm{eV}$, respectively [40], which means that the $\mathrm{SnSe}_{2}$ is more unstable than the $\mathrm{WSe}_{2}$ at the same condition. Moreover, these nanocomposited samples were prepared at $733 \mathrm{~K}$ under $60 \mathrm{MPa}$; this high temperature and pressure may provide a suitable environment for $\mathrm{SnSe}_{2}$ to react with InSb. Ulteriorly, EPMA was applied on the $\mathrm{SnSe}_{2}$ - and $\mathrm{WSe}_{2}$-composited samples, as shown in Fig. 2a and Fig. S2. The back-scattered electron (BSE) image and corresponding EDS characterizations indicate that the elements of In and $\mathrm{Sb}$ are spread evenly in the matrix except for 

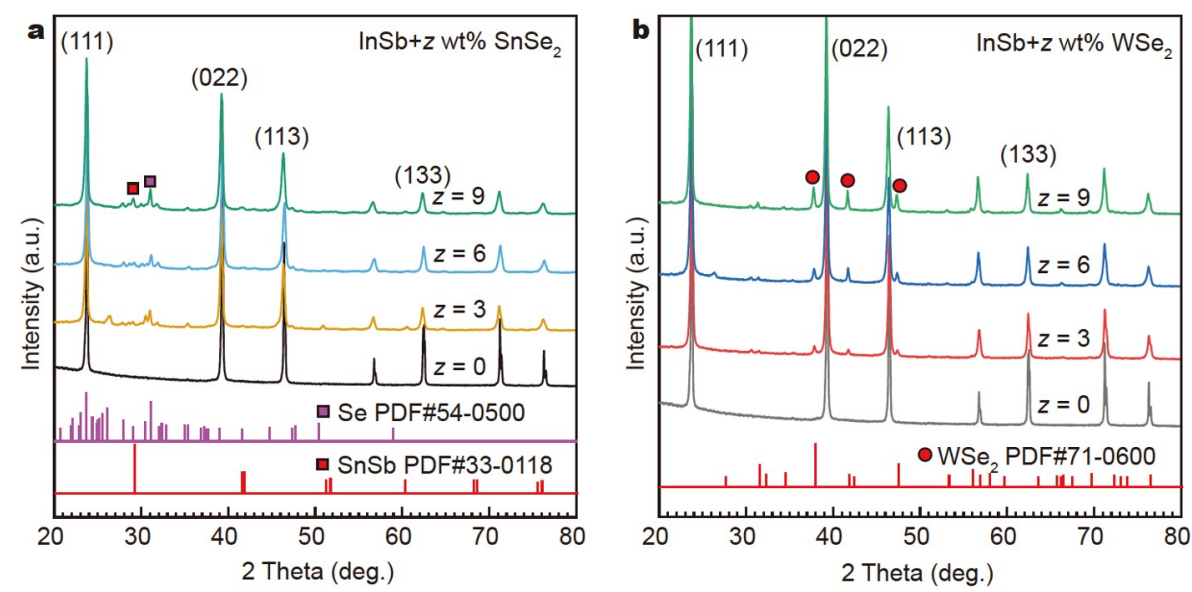

Figure 1 The room-temperature XRD patterns of (a) InSb $+z$ wt $\% \operatorname{SnSe}_{2}(z=0,3,6,9)$ and (b) InSb $+z$ wt $\%$ WSe $(z=0,3,6,9) \operatorname{SPS}$-sintered samples
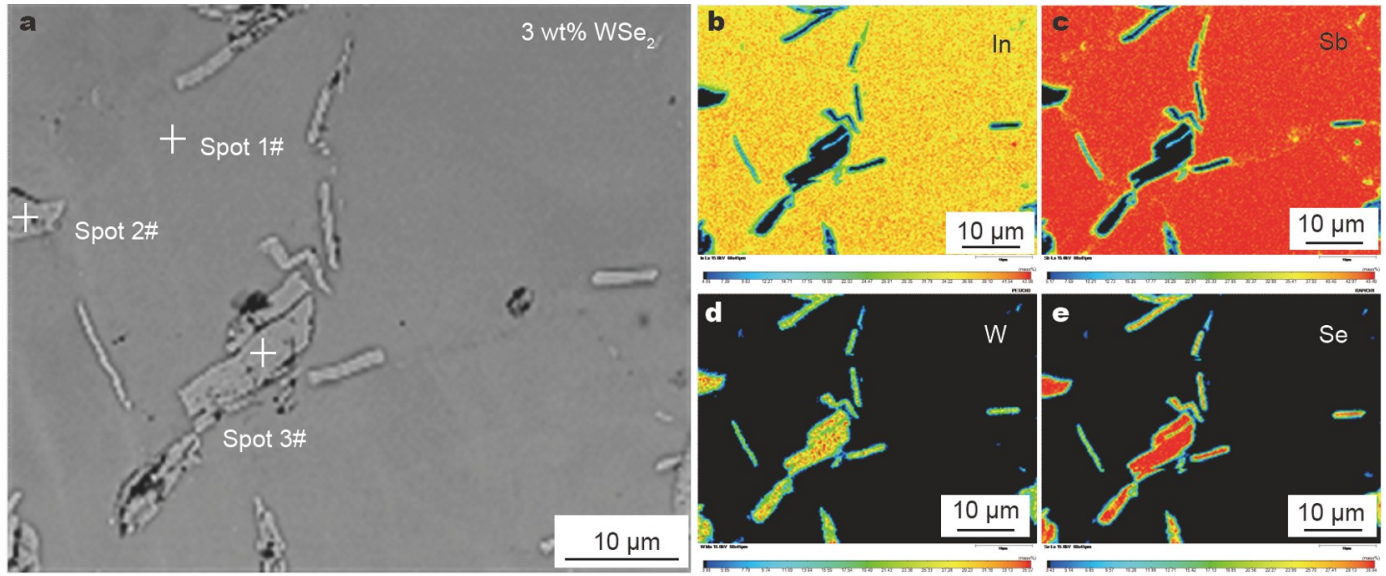

Figure 2 (a) The BSE electron pattern of $3 \% \mathrm{WSe}_{2}$ sample, (b-e) the corresponding EDS element mapping accompanied by the EPMA measurement.

Table 1 The EDS analysis of the spots marked in Fig. 2a

\begin{tabular}{|c|c|c|c|c|c|}
\hline Spots & $\mathrm{Se}$ & In & $\mathrm{Sb}$ & $\mathrm{W}$ & Se:W \\
\hline $1 \#$ & - & 49.75 & 50.25 & - & - \\
\hline 2\# & 58.50 & 0.68 & 0.98 & 39.84 & 1.47 \\
\hline 3\# & 57.47 & 1.52 & 1.44 & 39.58 & 1.45 \\
\hline
\end{tabular}

some fibrous $\mathrm{W}$ - and Se-rich regions. Moreover, the element analysis of area (Fig. 2b-e) and spots (Table 1) for the $3 \% \mathrm{WSe}_{2}$ sample manifests that these long $\mathrm{W}$ - and Se-rich inclusions are $\mathrm{WSe}_{2}$ phases, The ratio of Se:W is slightly lower than the intrinsic nominal $(\sim 2)$, indicating that part of Se and $\mathrm{W}$ may be dissolved into the InSb crystal.

To further explore the microstructure of $\mathrm{QSe}_{2}$-composited samples, we also adopted an advanced TEM technique to observe the $3 \% \mathrm{WSe}_{2}$ and $\mathrm{SnSe}_{2}$ samples in an HAADF mode, as shown in Fig. 3 and Fig. S3a, respectively. It can be seen that the $\mathrm{WSe}_{2}$ second phases were detected inside the composites (Fig. 3a). Moreover, a part of elongated fibrous $\mathrm{WSe}_{2}$ inclusions $(\sim 300 \mathrm{~nm})$ were embedded in the InSb matrix (Fig. 3b), a small amount of $\mathrm{W}$ and Se are uniformly dissolved in the matrix, and this can be formed as the $\mathrm{W}_{\mathrm{In}}{ }^{+}, \mathrm{Se}_{\mathrm{Sb}}{ }^{+} \mathrm{PDs}$ suchlike. Similarly, several inhomogeneous phases with different Z-contrasts can be clearly observed in the $\mathrm{SnSe}_{2}$ sample (Fig. S3a), and the corre- sponding EDS results reveal that these impurities are indeed $\mathrm{SnSb}$ and $\mathrm{Se}$, which is also consistent with the previous XRD measurement. The low-resolution TEM images in Fig. 4a, b and Fig. S3b, c affirm the ample hierarchical microstructures, and the inset of Fig. $4 \mathrm{a}$ is the corresponding selected area electron diffraction (SAED) pattern well indexed by the cubic InSb structure. A large amount of $\mathrm{WSe}_{2}$ nanofibers (Fig. $4 \mathrm{a}, \mathrm{b}$ ), abundant dislocation clusters (Fig. 4c) and $\mathrm{SnSb} / \mathrm{Se}$ nanoinclusions (Fig. S3b-d) germinated well in the InSb grains. Thereinto, the $\mathrm{WSe}_{2}$ generated a good heterogeneous interface with the matrix due to the stable nature of $\mathrm{WSe}_{2}$ and its congenial crystal formation energy with InSb. Strain simulation detailed by the geometric phase analysis (GPA) [41] was performed on the HRTEM image of Fig. 4d, which shows some drastically distorted regions between Grain A and Grain B. This can be directly revealed in the elastic strain map (inset of Fig. 4d) with aligned strain centers, facilitating an extensive phonon scattering 

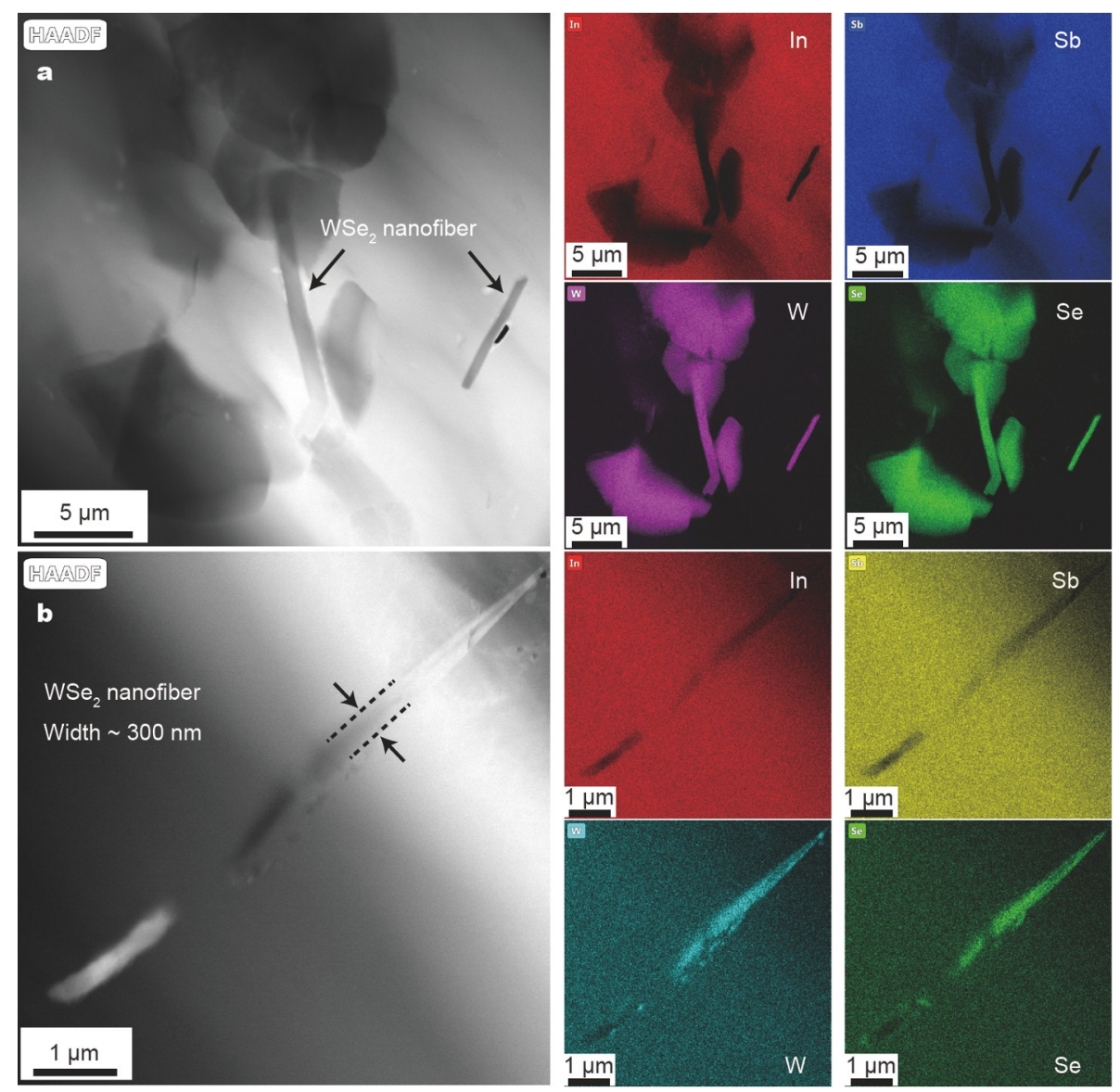

Figure $3(\mathrm{a}, \mathrm{b})$ The HAADF images of $3 \% \mathrm{WSe}_{2}$ sample and the corresponding EDS characterizations of surface scanning.

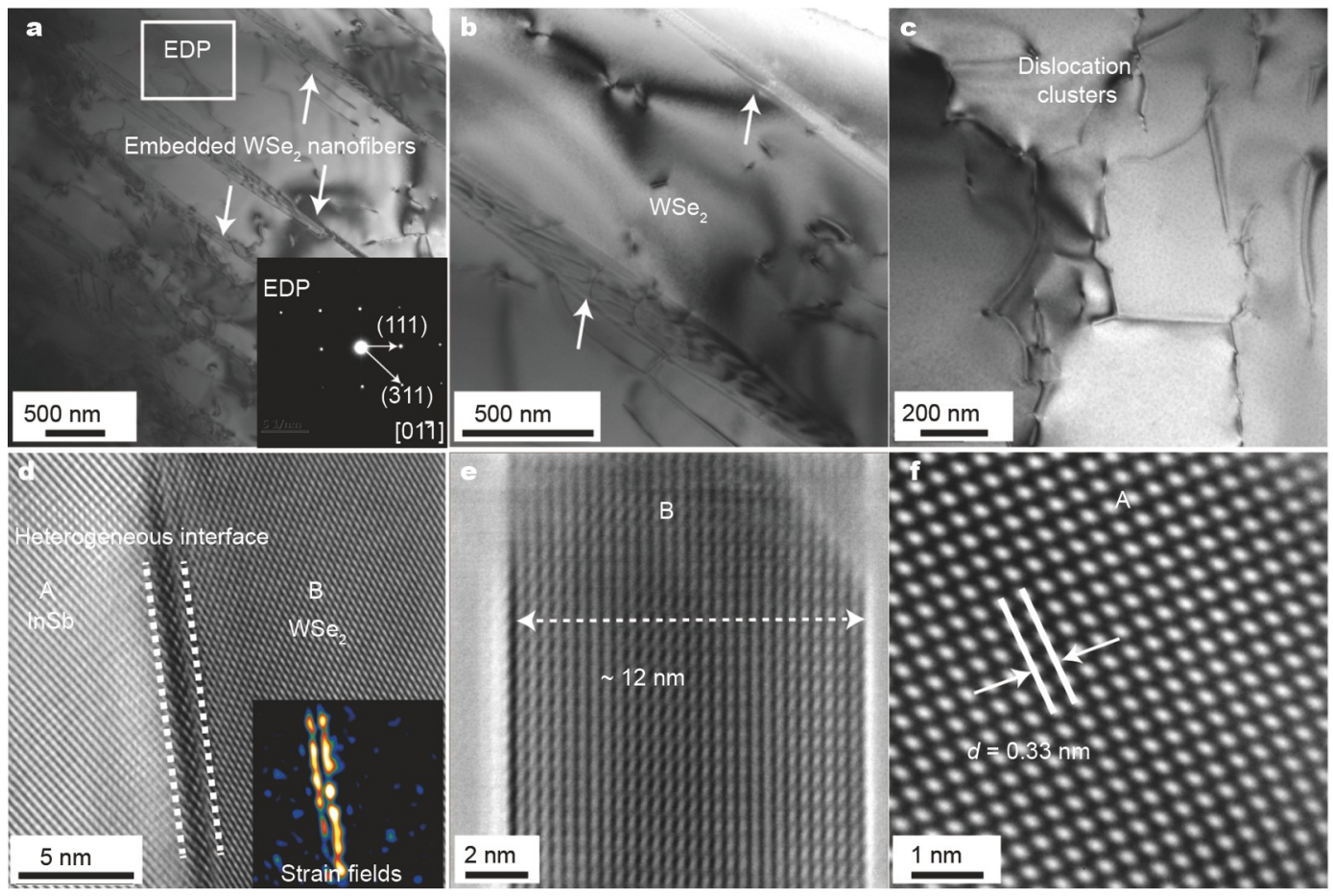

Figure 4 (a-c) The low-resolution TEM images of 3\% WSe $e_{2}$ sample with an inset of the SAED pattern in (a), displaying some embedded WSe $e_{2}$ nanofibers and abundant dislocation clusters. The HRTEM images of (d) heterogeneous interface. Inset is the corresponding GPA. (e) WSe $e_{2}$ nanostructure and (f) highly crystalline InSb matrix with the lattice spacing of $\sim 0.33 \mathrm{~nm}$. 
induced by a heterogeneous interface. Overall, these hierarchical structures, as well as the dispersed clusters of dislocations and dense stacking faults (SFs, Fig. S3e, f) combined with a number of distorted centers (marked in the inverse fast Fourier transform image), play an important role in multi-scale phonon propagation barriers in the electrical and thermal transport properties for the InSb system, and this will be discussed in the following sections.

\section{Electrical transport properties}

The electrical properties of $\mathrm{QSe}_{2}$-composited samples were displayed in Fig. 5, and the detailed properties of $z \mathrm{wt} \% \mathrm{SnSe}_{2}$ and $\mathrm{WSe}_{2}(z=0,3,6,9)$ samples are shown in Figs S4-S6. The electrical resistivity $\rho$ of $\mathrm{QSe}_{2}$ shows an obvious decrement with elevating temperature compared with the pristine InSb (Fig. 5a), which are both far lower than the ultra-high electrical resistivity of $\mathrm{WSe}_{2}$. Moreover, we conducted the Hall measurements as shown in Fig. 5b. It can be easily seen that the carrier concentrations of $\mathrm{QSe}_{2}$ are increased, even though the mobilities are lower than that of the InSb matrix. The significant enhancement of carrier concentration can be ascribed to the implants of Sn, W and Se observed in the microstructure characterizations for $\mathrm{QSe}_{2}$ samples. It should be noted that the concentration of nano $\mathrm{WSe}_{2}$ is very low, and thus the p-type carriers of intrinsic WSe $\mathrm{W}_{2}$ cannot compensate the largely enhanced carrier concentration induced by PDs. The Seebeck coefficient $|S|$ of $\mathrm{WSe}_{2}$-composited samples is larger than that of $\mathrm{SnSe}_{2}$ (Fig. 5c), and the calculated conduction and valence band [42-44] aligned at Fermi level
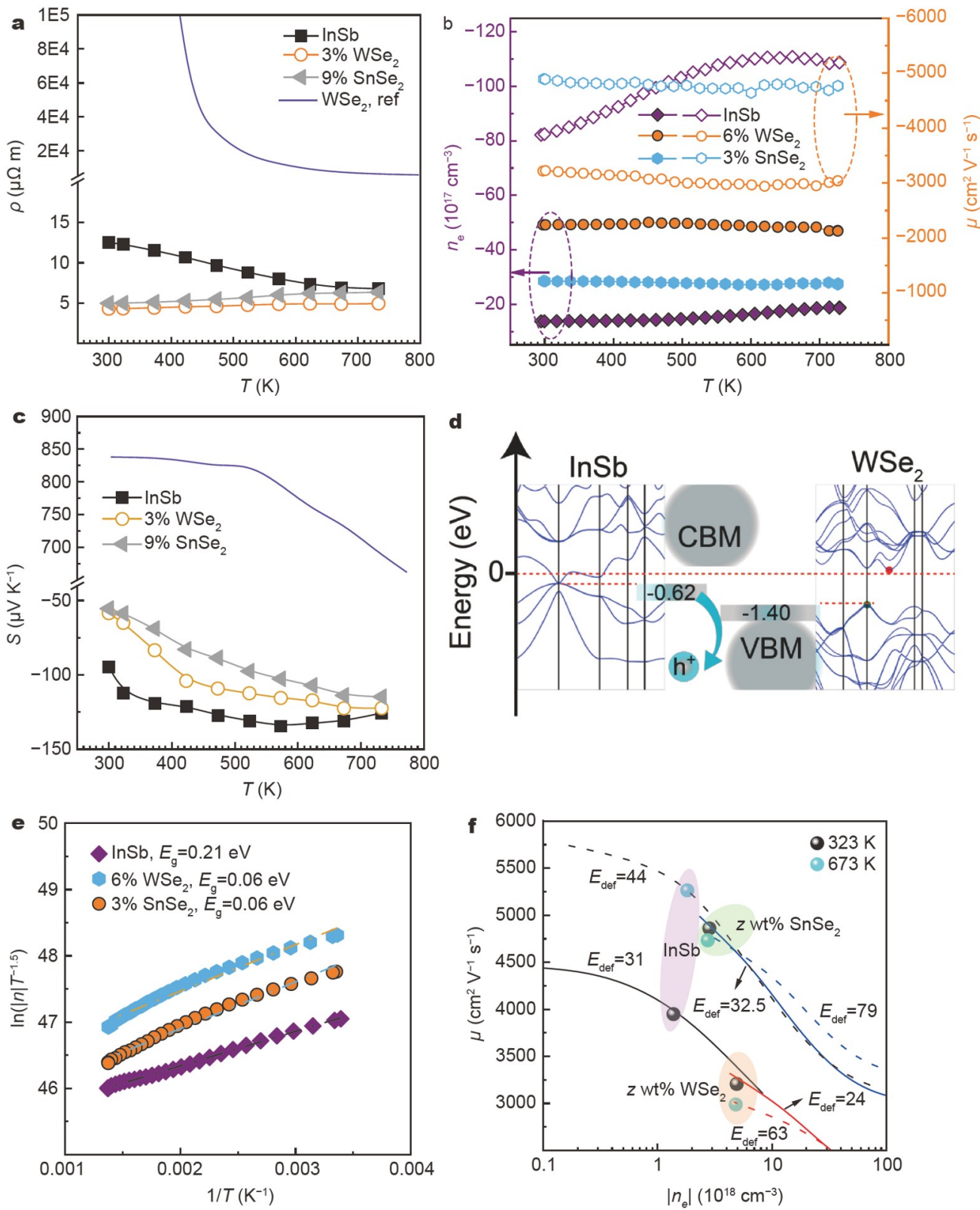

Figure 5 The temperature dependence of (a) electrical resistivity, (b) Hall measurement, (c) Seebeck coefficient for InSb and QSe $(\mathrm{Q}=\mathrm{Sn}, \mathrm{W})$ composites with the cited experimental results of $\mathrm{WSe}_{2}$ [36]. (d) The calculated energy structure of InSb matrix and WSe $e_{2}$. (e) The evaluated energy gap obtained by the equation $E_{\mathrm{g}}=K_{\mathrm{B}}{ }^{*} \ln \left(|n| T^{-1.5}\right) /\left(T^{-1}\right)$, where $K_{\mathrm{B}}, n$ is the Boltzmann constant and carrier concentration. (f) The deformation coefficient $E_{\text {def }}$ calculated from the two-band model compared to the experimental results. 
between InSb and $\mathrm{WSe}_{2}$ indicate that the valence band maximum $(\mathrm{VBM})$ of $\mathrm{WSe}_{2}(\sim-1.40 \mathrm{eV})$ is lower than that of the matrix ( $-0.62 \mathrm{eV})$ (Fig. $5 \mathrm{~d}$ ), which can effectively filter partial hole carriers, thus enhancing the whole Seebeck coefficient. With the measured Hall coefficient, the evaluated energy gap $E_{\mathrm{g}}$ values of InSb and $\mathrm{QSe}_{2}$ samples are displayed in Fig. 5e. The reduced energy gap $(\sim 0.06 \mathrm{eV})$ of composited samples is consistent with the increased carrier concentration induced by the $\mathrm{Q}$ and Se co-doping. Furthermore, based on the Kane energy model (Supplementary information Note 1), the deformation coefficient $\left(E_{\text {def }}\right)$ accompanied with the measured data for InSb and $\mathrm{QSe}_{2}$-composited samples are displayed in Fig. 5f. The $E_{\mathrm{def}}$ differences between 323 and $673 \mathrm{~K}$ such as $E_{\text {def }} @ I n S b$ from 31 to $44 \mathrm{eV}, E_{\mathrm{def}} @ \mathrm{SnSe}_{2}$ from 32.5 to $79 \mathrm{eV}$ and $E_{\mathrm{def}} @ \mathrm{WSe} \mathrm{e}_{2}$ from 24 to $63 \mathrm{eV}$, suggest that the scattering effect between electrons and phonons increases significantly with increasing temperature, which is beneficial for decreasing the lattice thermal conductivity.

\section{Thermal transport properties}

The total thermal conductivities $k_{\text {tot }}$ of $\mathrm{InSb}+z \mathrm{wt} \% \mathrm{QSe}_{2}(\mathrm{Q}=$ $\mathrm{Sn}, \mathrm{W})$ samples as a function of temperature are depicted in
Fig. 6a. The $k_{\text {tot }}$ of $9 \% \mathrm{SnSe}_{2}$ and $3 \% \mathrm{WSe}_{2}$ samples reduce significantly than the pristine InSb matrix; one of the reasons is the low intrinsic thermal conductivity of $\mathrm{WSe}_{2}\left(\sim 2.1 \mathrm{~W} \mathrm{~m}^{-1} \mathrm{~K}^{-1}\right.$ at $300 \mathrm{~K})$ as shown in Fig. $6 \mathrm{a}$, and on the other hand it is mainly because of the reductions in lattice thermal conductivity $k_{\text {lat }}$ thus leading to the decreased $k_{\text {tot }}$. More specifically, the $k_{\text {lat }}$ of InSb decreases from $\sim 14.1$ to $\sim 7.65 \mathrm{~W} \mathrm{~m}^{-1} \mathrm{~K}^{-1}$ in $3 \% \mathrm{WSe}_{2}$ and $\sim 7.65 \mathrm{~W} \mathrm{~m}^{-1} \mathrm{~K}^{-1}$ in $9 \% \mathrm{SnSe}_{2}$ at room temperature, $\sim 4.92$ to $\sim 2.73$ and $\sim 2.43 \mathrm{~W} \mathrm{~m}^{-1} \mathrm{~K}^{-1}$ at $673 \mathrm{~K}$, respectively. For better analyzing the possibilities of $k_{\text {lat }}$-reduction, the calculated $k_{\text {lat }}$ based on the well-known Callaway and two Kane band model (Supplementary information Note 2) with the consideration of various phonon scattering centers such as EP (electrons-phonons), GB (grain boundaries), PD, NP (nanoparticles) and SF are shown in Fig. 6b. The confirmed $\mathrm{W}_{\mathrm{In}}{ }^{+} / \mathrm{Se}_{\mathrm{Sb}}{ }^{+} \mathrm{PDs}$ and observed $\mathrm{WSe}_{2}$ nanosheets contribute most of the phonon scattering, thus significantly decreasing the $k_{\text {lat }}$. As presented in Fig. $6 c$, d, we further simulated the thermal transport and strain distribution (details refer to Supplementary information Note 3) with the comparison between nanofiber-based $\mathrm{WSe}_{2}$ and pristine InSb. Assuming that the ambient heat flow $(673 \mathrm{~K})$ is transferred from the hot-side to the cold-side of composites (Fig. 6c), the
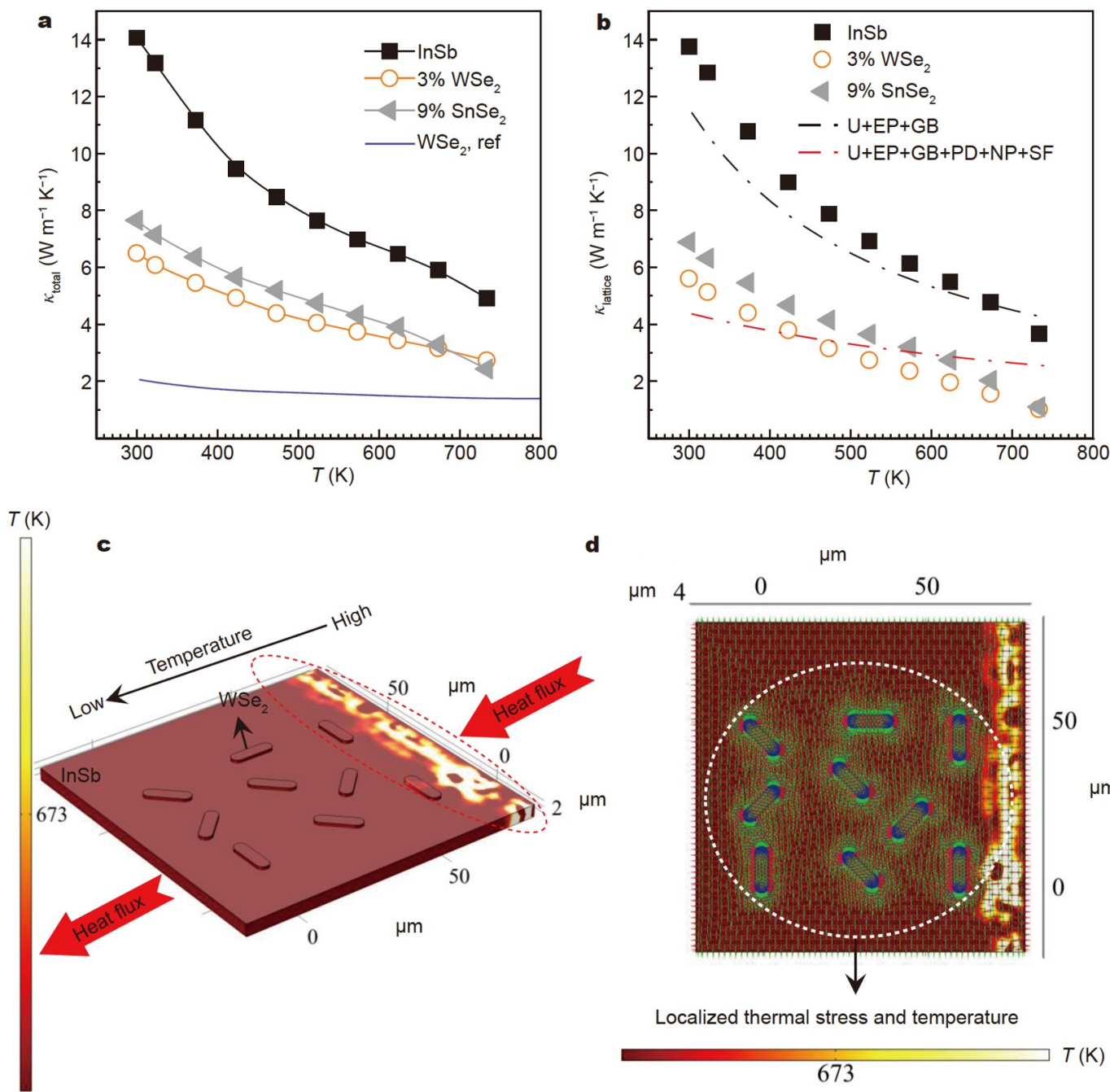

d

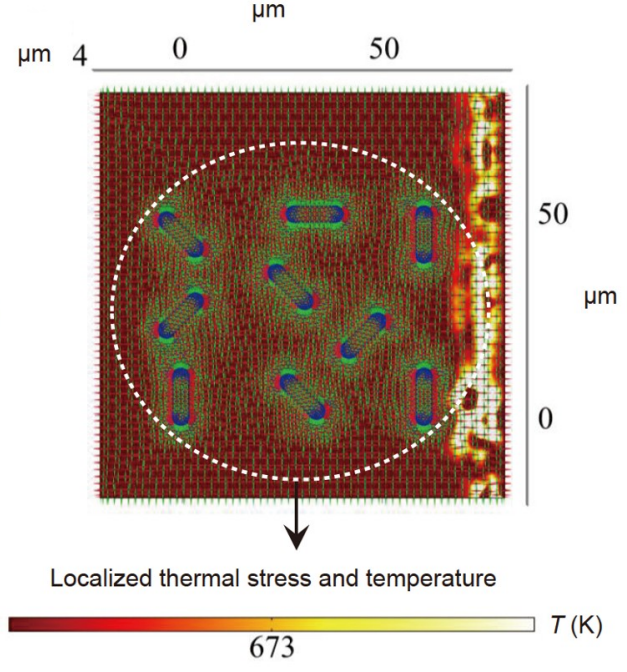

Figure 6 (a) The temperature-dependent total thermal conductivity $k_{\text {tot }}$ of InSb and $\mathrm{QSe} e_{2}(\mathrm{Q}=\mathrm{Sn}, \mathrm{W})$ composites. (b) The lattice thermal conductivity $k_{\text {lat }} v s$. temperature with the comparison of theoretical $k_{\text {lat }}$ obtained from the Callaway model. (c, d) The simulated thermal transport analysis of WSe $e_{2}$-composited samples based on the heat transfer and structural mechanics module in COMSOL Multiphysics software. 

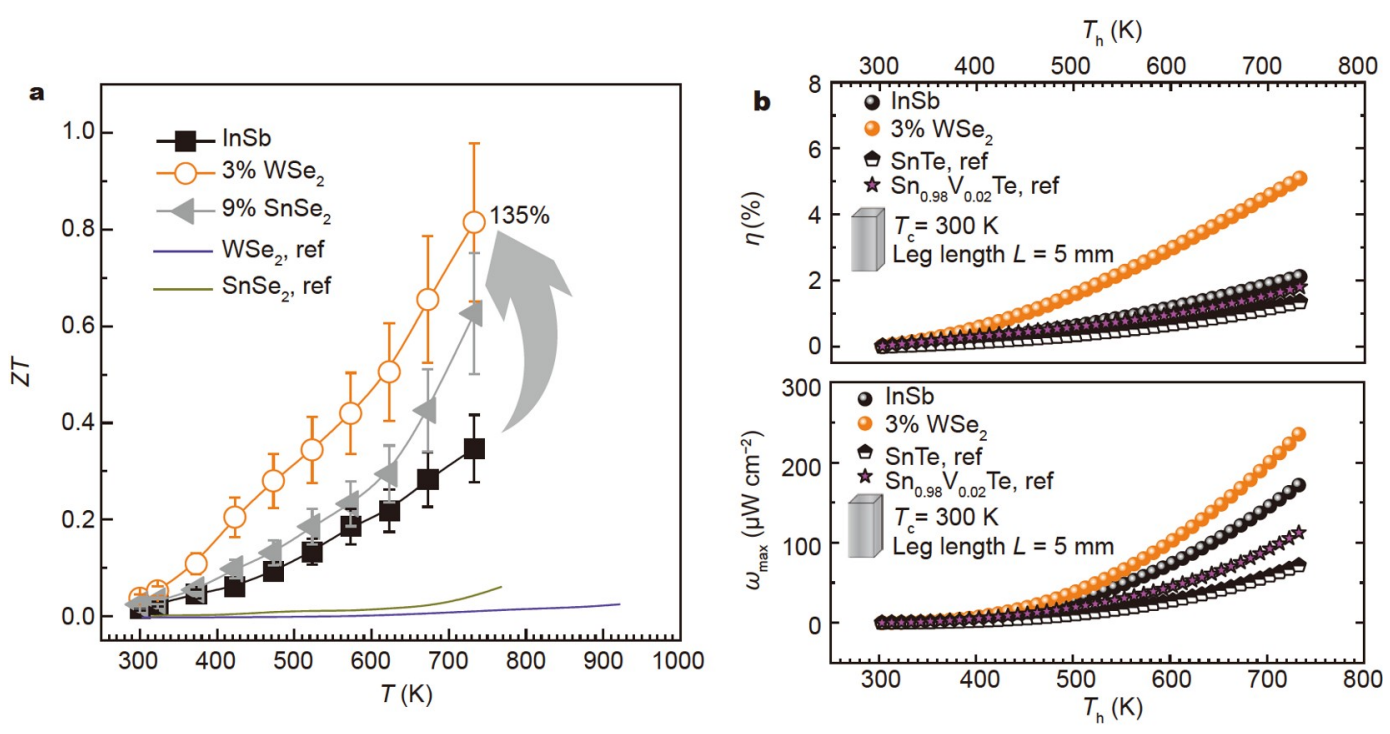

Figure 7 (a) The $Z T$ values $v s$. temperature for $\mathrm{QSe}_{2}$-composited InSb compared with the reported WSe $\mathrm{W}_{2}\left[36\right.$ ] and $\mathrm{SnSe}_{2}$ [35] systems. (b) The engineering output power density $\omega_{\max }$ and thermoelectric conversion efficiency $\eta \%$ dependent on hot-side temperature $T_{\mathrm{h}}$ assumed by the single thermoelectric leg length $L=5 \mathrm{~mm}$. The available mid-temperature SnTe thermoelectrics are included.

disorderly distributed $\mathrm{WSe}_{2}$ nanosheets acting as obstacles can effectively delay the heat flow diffusion due to the mismatch of thermal conductivity, which is advantageous for building the internal temperature difference in composites or thermoelectric devices. Moreover, the localized thermal stress and temperature (Fig. 6d) indicate that the nanosheet-based $\mathrm{WSe}_{2}$ disrupts the homogeneous thermal stress especially localized at the interface between $\mathrm{WSe}_{2}$ and InSb matrix, enhancing the effect on suppressing the propagation of phonons and reducing the lattice thermal conductivity. The corresponding heat transfer model of InSb (Fig. S7) shows anhysteretic heat conduction and uniform thermal stress distribution. Thus the heat flow can be quickly transferred from hot- to cold-side, which is also consistent with the experimental thermal transport properties with a high thermal conductivity.

Thermoelectric performance of $\mathrm{QSe}_{2}(\mathrm{Q}=\mathrm{Sn}, \mathrm{W})$ composites In view of the anionic-cationic PDs $\left(\mathrm{Q}_{\mathrm{In}}{ }^{+}, \mathrm{Se}_{\mathrm{Sb}}{ }^{+}\right)$and the favorable energy barrier between $\mathrm{WSe}_{2}$ composites and InSb matrix, the power factor of $6 \% \mathrm{WSe}_{2}$ sample (Fig. S6c) was significantly improved to $33.3 \mu \mathrm{W} \mathrm{cm} \mathrm{cm}^{-1} \mathrm{~K}^{-2}$ at $733 \mathrm{~K}$, with a $60.1 \%$ and $43 \%$ increment compared with $z \mathrm{wt} \% \mathrm{SnSe}_{2}$ composites (Fig. S5c) and pristine InSb, respectively. In addition, the abundant hierarchical microstructures such as heterogeneous interface and dislocations steadily reduce the total thermal conductivity and lattice thermal conductivity, thus enhancing the $Z T$ values for $z$ wt\% $\mathrm{QSe}_{2}$ composites as illustrated in Fig. 7a. The $Z T$ increases significantly from $\sim 0.35$ for InSb to $\sim 0.63$ for $9 \% \mathrm{SnSe}_{2}$ and $\sim 0.82$ for $3 \% \mathrm{WSe}_{2}$ at $733 \mathrm{~K}$, suggesting that the low-cost technology detailed by $\mathrm{QSe}_{2}$ nanocompositing can synergistically regulate the electrical and thermal properties and effectively enhance the thermoelectric performance. Besides, the evaluation of potential power generation based on the Thomson and Joule heat $[45,46]$ (Supplementary information Note 4) for optimized sample (3\% $\left.\mathrm{WSe}_{2}\right)$ was displayed in Fig. 7b, including a comparison to mid-temperature SnTe system [13] from 300 to $733 \mathrm{~K}$. The engineering output power density $\omega_{\max }$ of $\mathrm{WSe}_{2}$ nanocomposites increases to $\sim 235 \mu \mathrm{W} \mathrm{cm}{ }^{-2}$ at $733 \mathrm{~K}$, superior to pristine InSb as well as referred $\mathrm{Sn}_{0.98} \mathrm{~V}_{0.02} \mathrm{Te}$, enabling a thermoelectric conversion efficiency $\eta \sim 5.1 \%$ assumed by the single thermoelectric leg length $L \sim 5 \mathrm{~mm}$. These encouraging results reveal that the mid-temperature InSb-based thermoelectrics may possess a promising prospect in practical power generation with further reduction in thermal conductivity.

\section{CONCLUSIONS}

In this work, we have simultaneously regulated the electrical and thermal properties of InSb-based thermoelectrics in virtue of a low-cost and efficient nanocompositing of $\mathrm{QSe}_{2}(\mathrm{Q}=\mathrm{Sn}, \mathrm{W})$. More precisely, the improved electrical conductivity originated from the $\mathrm{QIn}^{+}$and $\mathrm{Se}_{\mathrm{Sb}}{ }^{+} \mathrm{PDs}$, since the deeper valence band of $\mathrm{WSe}_{2}$ is conductive to hindering these appreciate hole carriers, thus leading to a relatively increment of Seebeck coefficient when exceeding the intrinsic excitation temperature. Compared with $\mathrm{SnSe}_{2}$ composites and InSb, the enhanced power factor of $\sim 33.3 \mu \mathrm{W} \mathrm{cm} \mathrm{cm}^{-1} \mathrm{~K}^{-2}$ at $733 \mathrm{~K}$ was achieved in the $3 \% \mathrm{WSe}_{2}$ sample. As for thermal transports, the thermal conductivity and lattice thermal conductivity were thus significantly reduced due to the hierarchical microstructures such as PDs, nanosheets/ particles, and dislocations that constructed strong barriers to scatter multi-scale thermal phonons. Consequently, an enhanced $Z T$ value of $\sim 0.82$ at $733 \mathrm{~K}$ with a $135 \%$ improvement was obtained in $3 \% \mathrm{WSe}_{2}$ sample, in combination with the engineering output power density $\omega_{\max }$ of $\sim 233 \mu \mathrm{W} \mathrm{cm}{ }^{-2}$ and thermoelectric conversion efficiency $\eta$ of $\sim 5.2 \%$ in the single InSbbased thermoelectric leg. To sum up, motivated by selecting suitable compounds, nanocompositing can artificially merge effective strategies such as PDs, nanoengineering, and energy filtering into one, which may provide an ingenious way to optimize the thermoelectric properties of other thermoelectric systems.

Received 25 October 2021; accepted 2 December 2021; published online 24 January 2022

1 Xiao Y, Zhao LD. Seeking new, highly effective thermoelectrics. Sci- 
ence, 2020, 367: 1196-1197

2 Roychowdhury S, Ghosh T, Arora R, et al. Enhanced atomic ordering leads to high thermoelectric performance in $\mathrm{AgSbTe}_{2}$. Science, 2021, 371: 722-727

3 Shi XL, Zou J, Chen ZG. Advanced thermoelectric design: From materials and structures to devices. Chem Rev, 2020, 120: 7399-7515

4 Xin J, Basit A, Li S, et al. Inorganic thermoelectric fibers: A review of materials, fabrication methods, and applications. Sensors, 2021, 21: 3437

5 Dhawan $\mathrm{R}$, Madusanka $\mathrm{P}, \mathrm{Hu} \mathrm{G}$, et al. $\mathrm{Si}_{0.97} \mathrm{Ge}_{0.03}$ microelectronic thermoelectric generators with high power and voltage densities. Nat Commun, 2020, 11: 1

6 Qiu J, Yan Y, Xie H, et al. Achieving superior performance in thermoelectric $\mathrm{Bi}_{0.4} \mathrm{Sb}_{1.6} \mathrm{Te}_{3.72}$ by enhancing texture and inducing highdensity line defects. Sci China Mater, 2021, 64: 1507-1520

7 Heremans JP, Jovovic V, Toberer ES, et al. Enhancement of thermoelectric efficiency in $\mathrm{PbTe}$ by distortion of the electronic density of states. Science, 2008, 321: 554-557

8 Zeier WG, Schmitt J, Hautier G, et al. Engineering half-Heusler thermoelectric materials using Zintl chemistry. Nat Rev Mater, 2016, 1: 1

9 Chen ZG, Shi X, Zhao LD, et al. High-performance SnSe thermoelectric materials: Progress and future challenge. Prog Mater Sci, 2018, 97: 283346

10 Gao Z, Yang Q, Qiu P, et al. p-type plastic inorganic thermoelectric materials. Adv Energy Mater, 2021, 11: 2100883

11 Zhang J, Zhang T, Zhang H, et al. Single-crystal SnSe thermoelectric fibers via laser-induced directional crystallization: From 1D fibers to multidimensional fabrics. Adv Mater, 2020, 32: 2002702

12 Luo Y, Cai S, Hao S, et al. High-performance thermoelectrics from cellular nanostructured $\mathrm{Sb}_{2} \mathrm{Si}_{2} \mathrm{Te}_{6}$. Joule, 2020, 4: 159-175

13 Xin J, Li S, Yang J, et al. Tactfully decoupling interdependent electrical parameters via interstitial defects for SnTe thermoelectrics. Nano Energy, 2020, 67: 104292

14 Xin J, Yang J, Jiang Q, et al. Reinforced bond covalency and multiscale hierarchical architecture to high performance eco-friendly MnTe-based thermoelectric materials. Nano Energy, 2019, 57: 703-710

15 He W, Wang D, Wu H, et al. High thermoelectric performance in lowcost $\mathrm{SnS}_{0.91} \mathrm{Se}_{0.09}$ crystals. Science, 2019, 365: 1418-1424

16 Tang J, Gao B, Lin S, et al. Manipulation of band structure and interstitial defects for improving thermoelectric SnTe. Adv Funct Mater, 2018, 28: 1803586

17 Ma Z, Xu T, Li W, et al. High entropy semiconductor AgMnGeSbTe with desirable thermoelectric performance. Adv Funct Mater, 2021, 31: 2103197

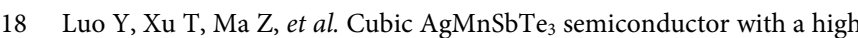
thermoelectric performance. J Am Chem Soc, 2021, 143: 13990-13998

19 Jiang Q, Li S, Luo Y, et al. Ecofriendly highly robust $\mathrm{Ag}_{8} \mathrm{SiSe}_{6}$-based thermoelectric composites with excellent performance near room temperature. ACS Appl Mater Interfaces, 2020, 12: 54653-54661

20 Xin J, Yang J, Li S, et al. Thermoelectric performance of rapidly microwave-synthesized a-MgAgSb with SnTe nanoinclusions. Chem Mater, 2019, 31: 2421-2430

21 Chen Z, Ge B, Li W, et al. Vacancy-induced dislocations within grains for high-performance PbSe thermoelectrics. Nat Commun, 2017, 8: 13828

22 Chen Z, Jian Z, Li W, et al. Lattice dislocations enhancing thermoelectric PbTe in addition to band convergence. Adv Mater, 2017, 29: 1606768

23 Li S, Xin J, Basit A, et al. In situ reaction induced core-shell structure to ultralow $\kappa_{\text {lat }}$ and high thermoelectric performance of SnTe. Adv Sci, 2020, 7: 1903493

24 Jiang Q, Hu H, Yang J, et al. High thermoelectric performance in SnTe nanocomposites with all-scale hierarchical structures. ACS Appl Mater Interfaces, 2020, 12: 23102-23109

25 Vurgaftman I, Meyer JR, Ram-Mohan LR. Band parameters for III-V compound semiconductors and their alloys. J Appl Phys, 2001, 89: 5815-5875

26 Massidda S, Continenza A, Freeman AJ, et al. Structural and electronic properties of narrow-band-gap semiconductors: InP, InAs, and InSb.
Phys Rev B, 1990, 41: 12079-12085

27 Su XL, Tang XF, Li H. Effects of melt spinning process on microstructure and thermoelectric properties of n-type InSb compounds. Acta Physica Sinica, 2010, 4: 2860-2866

28 Zhang Q, Xiong Z, Jiang J, et al. Enhanced thermoelectric performance in $\mathrm{In}_{1-x} \mathrm{Ga}_{x} \mathrm{Sb}$ originating from the scattering of point defects and nanoinclusion. J Mater Chem, 2011, 21: 12398

29 Jiang G, Chen Y, Zhu T, et al. Microstructure and thermoelectric properties of InSb compound with nonsoluble NiSb in situ precipitates. J Mater Res, 2013, 28: 3394-3400

30 Xin J, Jiang Q, Wen Y, et al. An in situ eutectic remelting and oxide replacement reaction for superior thermoelectric performance of InSb. J Mater Chem A, 2018, 6: 17049-17056

31 Cheng Y, Yang J, Jiang Q, et al. New insight into InSb-based thermoelectric materials: From a divorced eutectic design to a remarkably high thermoelectric performance. J Mater Chem A, 2017, 5: 5163-5170

32 Jang H, Park JH, Lee HS, et al. Regulating Te vacancies through dopant balancing via excess Ag enables rebounding power factor and high thermoelectric performance in p-type PbTe. Adv Sci, 2021, 8: 2100895

33 Zhao W, Liu Z, Sun Z, et al. Superparamagnetic enhancement of thermoelectric performance. Nature, 2017, 549: 247-251

34 Luo Y, Yang J, Jiang Q, et al. Progressive regulation of electrical and thermal transport properties to high-performance $\mathrm{CuInTe}_{2}$ thermoelectric materials. Adv Energy Mater, 2016, 6: 1600007

35 Luo Y, Zheng Y, Luo Z, et al. n-type $\mathrm{SnSe}_{2}$ oriented-nanoplate-based pellets for high thermoelectric performance. Adv Energy Mater, 2018, 8: 1702167

36 Liu Y, Liu J, Tan X, et al. High-temperature electrical and thermal transport behaviors in layered structure $\mathrm{WSe}_{2}$. J Am Ceram Soc, 2017, 100: 5528-5535

37 Kresse G, Joubert D. From ultrasoft pseudopotentials to the projector augmented-wave method. Phys Rev B, 1999, 59: 1758-1775

38 Zhao W, Liu Z, Wei P, et al. Magnetoelectric interaction and transport behaviours in magnetic nanocomposite thermoelectric materials. Nat Nanotech, 2017, 12: 55-60

39 Xiao $\mathrm{Y}, \mathrm{Wu} \mathrm{H}$, Wang $\mathrm{D}$, et al. Amphoteric indium enables carrier engineering to enhance the power factor and thermoelectric performance in n-type $\mathrm{Ag}_{n} \mathrm{~Pb}_{100} \mathrm{In}_{n} \mathrm{Te}_{100+2 n}$ (LIST). Adv Energy Mater, 2019, 9: 1900414

40 Jain A, Ong SP, Hautier G, et al. Commentary: The materials project: A materials genome approach to accelerating materials innovation. APL Mater, 2013, 1: 011002

41 Hÿtch MJ, Snoeck E, Kilaas R. Quantitative measurement of displacement and strain fields from HREM micrographs. Ultramicroscopy, 1998, 74: 131-146

42 Vergniory MG, Wieder BJ, Elcoro L, et al. All topological bands of all stoichiometric materials. arXiv: 2105.09954, 2021

43 Vergniory MG, Elcoro L, Felser C, et al. A complete catalogue of highquality topological materials. Nature, 2019, 566: 480-485

44 Bradlyn B, Elcoro L, Cano J, et al. Topological quantum chemistry. Nature, 2017, 547: 298-305

45 Guo $\mathrm{F}, \mathrm{Wu} \mathrm{H}$, Zhu J, et al. Synergistic boost of output power density and efficiency in In-Li-codoped SnTe. Proc Natl Acad Sci USA, 2019, 116: 21998-22003

46 Liu W, Kim HS, Chen S, et al. n-type thermoelectric material $\mathrm{Mg}_{2} \mathrm{Sn}_{0.75} \mathrm{Ge}_{0.25}$ for high power generation. Proc Natl Acad Sci USA, 2015, 112: 3269-3274

Acknowledgements This work was supported by the National Natural Science Foundation of China (92163211 and 51872102), Foshan (Southern China) Institute for New Materials (2021AYF25005), Singapore Ministry of Education Academic Research Fund Tier 2 (MOE2019-T2-2-127 and MOET2EP50120-0002), the A*STAR under AME IRG (A2083c0062), Singapore Ministry of Education Academic Research Fund Tier 1 (RG90/19 and RG73/ 19), and Singapore National Research Foundation Competitive Research Program (NRF-CRP18-2017-02). The technical assistance from the Analytical and Testing Center of HUST and Wuhan University of Technology are likewise gratefully acknowledged. 
Author contributions Xin J designed this project; Xin J, Li W, Li S and Tao $\mathrm{Y}$ conducted the experiments; Xu T performed the EPMA analyses on the samples; Jiang Q and Luo Y participated in the discussion of the experimental results; Wei L and Yang J revised this manuscript. All authors have discussed and approved the results and conclusions of this article.

Conflict of interest The authors declare that they have no conflict of interest.

Supplementary information Experimental details and supporting data are available in the online version of the paper.

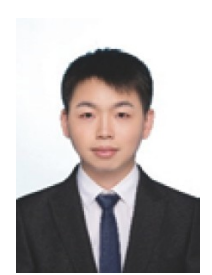

Jiwu Xin is currently a research fellow of the School of Electrical and Electronic Engineering at Nanyang Technological University, Singapore. He received his BS degree from the School of Materials Science and Engineering, Anhui University of Technology, China in 2015, and obtained his $\mathrm{PhD}$ degree from Huazhong University of Science and Technology, China in 2020. His research interests focus on thermoelectric materials, and flexible and wearable functional materials.

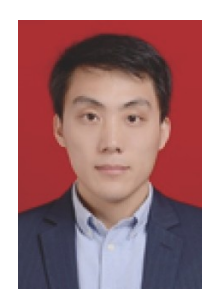

Yubo Luo is currently a professor at Huazhong University of Science and Technology, China. He received his BS degree in materials physics in 2011 from Yan-Shan University, China, and his $\mathrm{PhD}$ degree from Huazhong University of Science and Technology, China, in 2016. He was a research fellow at Nanyang Technological University, Singapore (2016-2019), and a visiting scholar at Northwestern University, United States (2017-2019). His current research focuses on thermoelectric semiconductors.

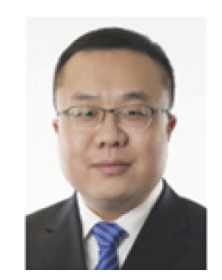

Lei Wei is an associate professor at Nanyang Technological University in Singapore. He received the BE degree from Wuhan University of Technology in 2005, and the PhD degree from the Technical University of Denmark in 2011. Then he joined Massachusetts Institute of Technology as a postdoctoral associate. In 2014, he joined the School of Electrical and Electronic Engineering at Nanyang Technological University in Singapore as a Nanyang assistant professor. In 2019, he was promoted to associate professor with tenure. His main research interests are fiber-based devices, multi-functional fibers, bio-fiber interfaces, and in-fiber energy generation and storage.

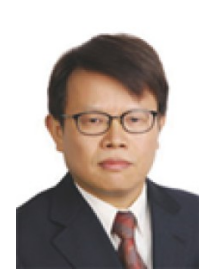

Junyou Yang is currently deputy dean of the Department of Materials Science, School of Materials Science and Engineering at Huazhong University of Science and Technology, China. He obtained his $\mathrm{PhD}$ degree from Huazhong University of Science and Technology, China in 1996. He was a postdoctor at the Research Center of Advanced Science and Technology (RCAST), University of Tokyo, Japan (1999-2001). He specializes in thermoelectric materials, fusion functional materials, and solar cells.

\section{通过二维层状结构搭建能量和声子势垒提高InSb热 电性能}

辛集武 ${ }^{1}$, 李旺 ${ }^{1}$, 李思慧 ${ }^{1}$ 陶阳 ${ }^{1}$, 许天 ${ }^{1}$, 罗裕波 ${ }^{*}$, 姜庆辉 ${ }^{1}$, 魏磊 ${ }^{*}$, 杨君友 ${ }^{*}$

摘要 $\mathrm{InSb}$ 是一种具有闪锌矿结构的窄带隙半导体, 在光电探测器、红 外热成像、霍尔器件等领域有着广泛的应用. 良好的能带结构、超高 的电子迁移率和无毒性质表明 InSb 可能是一种潜在的中温热电材料. InSb 当前面临着一些关键挑战, 如高导热系数和小Seebeck系数, 导致其 超高的晶格导热系数, 进而低的 $Z T$ 值. 鉴于此, 我们发展了一种 $z w \mathrm{t} \%$ $\mathrm{QSe}_{2}(\mathrm{Q}=\mathrm{Sn}, \mathrm{W})$ 纳米复合的高效策略, 即通过对二维层状 $\mathrm{QSe}_{2}$ 的纳米 复合, 在 $\mathrm{InSb}$ 体系中引入 $\mathrm{Q}_{\mathrm{In}}{ }^{+}$和 $\mathrm{Se}_{\mathrm{Sb}}{ }^{+}$点缺陷. 此外, 本征WSe ${ }_{2}$ 的价带作 为势垒可以散射相当数量的空穴载流子, 导致本征激发阶段的Seebeck 系数升高. 此外, 无序分布的纳米片/粒子和位错可以有效地阻碍热流 扩散, 形成热声子的强散射. 结果表明, $3 \% \mathrm{WSe}_{2}$ 样品的功率因子提高到 $\sim 33.3 \mu \mathrm{W} \mathrm{cm}{ }^{-1} \mathrm{~K}^{-2}, 733 \mathrm{~K}$ 时 $Z T$ 值提高到 $\sim 0.82$, 工程输出功率密度 $\omega_{\text {max }}$ $\sim 233 \mu \mathrm{W} \mathrm{cm}{ }^{-2}$, 工程热电转换效率 $\eta \sim 5.2 \%$. 